\title{
Educación ambiental mediante la investigación como estrategia pedagógica ${ }^{1}$ \\ Environmental education through research as a pedagogical strategy
}

DOI: http://dx.doi.org/10.17981/cultedusoc.9.1.2018.18

Fecha de recepción: 18/05/2018. Fecha de aceptación: 21/08/2018

\section{María Vásquez-Thorné2 (iD}

Yarleidis Núñez-Sarmiento; Digna Hernández-Mejía; Senia Abello-Arambul; Katherine De Lima-Rodríguez; Danielly Cantillo-Jiménez; Marly Erazo-Rudas; Egracielys Bustamante-Bravo; Arles Aguilar-Retamozo; Indira Gómez-Martínez; Helmo Juvinao; Julián Campo; Mavis Granados-Lobo; Hortensia Márquez-Rodríguez; Manuel De León-Ortega; Edilsa Gámez-Cabarca; Zeila Rudas-Salamanca; Fernando Díaz-Herrera; María Arias-López; María Acosta-Herrera ${ }^{3}$

Para citar este artículo

Vásquez-Thorné, M., Núñez-Sarmiento, Y., Hernández-Mejía, D., Abello-Arambul, S., De Lima-Rodríguez, K., Cantillo-Jiménez, D., Erazo-Rudas, M., Bustamante-Bravo, E., Aguilar-Retamozo, A., Gómez-Martínez, I., Juvinao, H., Campo, J., Granados-Lobo, M., Márquez-Rodríguez, H., De León-Ortega, M., Gámez-Cabarca, E., Rudas-Salamanca, Z., Díaz-Herrera, F., Arias-López, M. y Acosta-Herrera, M. (2018). Educación ambiental mediante la investigación como estrategia pedagógica. Cultura. Educación y Sociedad 9(1), 228-239. DOI: http://dx.doi.org/10.17981/cultedusoc.9.1.2018.18

\section{Resumen}

La educación ambiental es una herramienta teóricopráctica que permite la integración de elementos sociales propios del contexto escolar, creando una ciudadanía consciente. El estudio busco, promover la educación ambiental mediante la investigación como estrategia pedagógica, guiado bajo los lineamientos metodológicos de la IEP con un diseño basado en las trayectorias de indagación, se contó con ochenta (80) participantes de la institución educativa Tucurinca ubicada en el Municipio de Zona Bananera Magdalena. Los resultados dejaron en evidencia diferentes problemáticas ambientales observadas por los estudiantes en la comunidad mediante el proceso de indagación, donde se encontró deterioro del humedal, proliferación de insectos y hongos producidos por basuras en descomposición que afectan a la salud de la población, para esto los estudiantes se organizaron en grupos de investigación y diseñaron propuestas para dar solución a los diferentes problemas encontrados, finalmente estas propuestas fueron divulgadas y socializadas en la feria institucional de la ciencia.

Palabras clave: educación ambiental, investigación como estrategia pedagógica, proyectos productivos, institución educativa.

\begin{abstract}
Environmental education is a theoretical-practical tool that allows the integration of social elements typical of the school context, creating a conscious citizenship. The study sought to promote environmental education through research as a pedagogical strategy, guided by the methodological guidelines of the IEP with a design based on the trajectories of inquiry, was attended by eighty (80) participants from the educational institution Tucurinca located in the Municipality of Magdalena Bananera Zone. The results showed different environmental problems observed by the students in the community through the process of investigation, where the wetland deterioration was found, as well as the proliferation of insects and fungi produced by decomposing garbage that affect the health of the population. students were organized in research groups and designed proposals to solve the different problems found, finally these proposals were disseminated and socialized in the institutional science fair.
\end{abstract}

Keywords: environmental education, research as a pedagogical strategy, productive projects, educational institution.

\footnotetext{
${ }^{1}$ Este artículo ha sido derivado del Programa de Fortalecimiento de la Cultura Ciudadana y Democrática CT+I a través de la IEP apoyada en TIC en el Departamento de Magdalena: CICLON

${ }^{2}$ Docente de la Institución Educativa Tucurinca, Sede principal y líder del grupo de investigación Eco investigadores 2.0. Correo de correspondencia: kelysgaviria@hotmail.com

${ }^{3}$ Docentes de la Institución Educativa Tucurinca, Sede principal y miembros del grupo de investigación Eco investigadores 2.0.
} 


\section{Introducción}

Las disciplinas científicas no han sido ajenas a la preocupación ambiental internacional, aumentándose el estudio, discusión y aproximaciones sistemáticas a la relación entre el comportamiento humano y el estado del ambiente con el propósito de contribuir a la explicación y comprensión de la problemática ambiental y a la búsqueda de soluciones. Para el caso de las ciencias humanas, su interés ha estado en el estudio de la responsabilidad que tienen los sistemas sociales y culturales sobre buena parte de los problemas ambientales, dado que el comportamiento contextualizado socialmente de las personas y su impacto en el medio ambiente (comportamientos ambientalmente relevantes) es una de las variables fundamentales para resolver eficientemente este tipo de problemas, así como para preservar los recursos y prevenir otros problemas ambientales.

El compromiso para contrarrestar el impacto ciudadano común sobre el ambiente se ha fijado en la educación ambiental como esfuerzo internacional y multisectorial adoptado por los ministerios de educación nacional de la región latinoamericana. En Colombia, se reglamentó con la Política Nacional de Educación Ambiental documento legal macro que señala los actores, instituciones, roles y metas de la educación ambiental. (Organización de las Naciones Unidas, 2002)

Aunque el acuerdo internacional sobre la importancia de la educación ambiental ha sido ampliamente respaldado no existe un único acercamiento sobre la forma en que debe aplicarse, ni de las acciones puntuales que se esperan de los actores educativos. Por el contrario, los países, sectores sociales y actores educativos presentan diferentes metas res- pecto al propósito final de la educación ambiental, así como los acercamientos didácticos, epistemológicos y económicos que debería tener, y de los indicadores de efectividad pedagógica resultado de su implementación (Sandoval, 2012).

Hoy, la crisis socio-ambiental ha alcanzado tal magnitud, que escapa del control humano; así, por primera vez en la historia de la humanidad se pone en peligro la vida en el planeta (Novo, 2006). Entre las causas por los cuales se entra en una crisis socio-ambiental se resaltan: (a) el papel de los seres humanos en la naturaleza ha sido y es muy destructivo; (b) la idea aristotélica de lo humano como centro, separado de lo natural; (c) el concepto antropocéntrico en la relación especie humana-naturaleza; (d) la relación humana con la biosfera, que a partir de la revolución urbano industrial, genera tremendos cambios en la cultura occidental, estimulando impactos negativos sobre el ambiente natural y (e) la visión mercado-céntrica en la relación sociedad-naturaleza.

Desde hace más de 500 años el modelo de desarrollo occidental (eurocentrista) con sus características -monoteísmo, racionalismo, patriarcalismo, capitalismo, antropocentrismo y ahora el mercado - se ha impuesto al resto de culturas y pueblos en el mundo. Donde la actual globalización neoliberal estimula la polarización entre países y sectores ricos y pobres, y acentúa, aún más, la brecha (económica, tecnológica, comercial, productiva) en los ámbitos nacional e internacional (Martínez, 2009).

La educación ambiental se ha constituido en un tema de obligatoria inclusión en las agendas públicas, de igual forma, los proyectos educativos institucionales (PEI), agendas ambientales, reuniones de gremios de la producción y sociedad 
civil en general. Es importante tener en cuenta lo ambiental como un tópico en el ámbito educativo, lo cual debe ser analizado en profundidad y manejado de tal manera que garantice en el mediano plazo un cambio en forma en que la sociedad se relaciona con su entorno y produce los bienes y servicios para colmar las necesidades de la comunidad en general.

Mantener buenas relaciones en el interior del sistema sociocultural, y con el sistema natural es un reto, pero también una necesidad que nos permite permanecer en el tiempo y en el espacio como especie, respetando el derecho a la existencia de los otros sistemas vivos, de igual manera, es indispensable cambiar el repertorio de pautas con los cuales nos interrelacionamos con el universo y recurrir al cambio actitudinal que logre dar nuevos valores a la vida como proceso y derecho de digno disfrute.

La IED Tucurinca busca educar e inducir a las próximas generaciones en la investigación científica sobre el cuidado y conservación del medio ambiente, proporcionando a los estudiantes espacios para trabajar en la búsqueda de soluciones a problemas ambientales y socioculturales de su entorno escolar, procurando garantizar bienestar y posibilitar una mejor calidad de vida; utilizando la ruta metodológica de la IEP que implica; indagación, innovación y herramientas TIC para así fomentar una cultura ciudadana y democrática para la apropiación del conocimiento.

La educación ambiental se convierte en una corriente que rompe con los esquemas tradicionales de enseñanza, orientado hacia modelos constructivistas, dando lugar a diferentes sustentos teóricos como; aprendizaje basado en problemas, trabajo sobre proyectos, aprendizaje por indagación, cooperativo, significativo y en contexto, (Perales, \& Ayerbe, 2016). Lo anterior permite desarrollar nuevas capacidades en los estudiantes promoviendo una educación integral de calidad que favorezca el proceso enseñanza-aprendizaje y dinamice los contenidos curriculares.

Es frecuente observar en los alrededores de la IED Tucurinca, la presencia de basuras arrojadas de manera indiscriminada, puesto que, no cuenta con un basurero y retiro del mismo, sumado a la poca cultura ambiental, que conlleva quema de basura, es por ello que la ornamentación, cuidado y conservación del medio ambiente a través de la implementación de proyectos productivos en la institución, permitirá el empoderamiento de actividades ambientales y conductas sostenibles para propender entornos saludables.

Actualmente existe una preocupación por el deterioro ambiental producido por actividades humanas, por ende, desde las instituciones educativas se busca impartir la educación ambiental con la finalidad de promover conciencia ciudadana, mediante la implementación de estrategias pedagógicas desde modelos desarrollistas, donde el estudiante tome un rol activo en la construcción de su conocimiento, por tal razón el estudio tuvo como objetivo promover la educación ambiental mediante proyectos productivos en la institución educativa Tucurinca de Zona Bananera.

Otro problema que se evidencia es el relacionado con el uso del agua potable por ser abastecidos del rio Tucurinca y sin efectuar ningún tipo de tratamiento que haga más saludable para la comunidad la utilización del preciado líquido y por el contrario se convierte en un foco de enfermedades debido al uso inadecuado. La falta de concienciación hacia la importancia de la separación de los residuos 
sólidos que a diario se originan en la comunidad a pesar de toda la formación que se les brinda e insiste en la utilización incluso de medios para facilitar estas diferencias como son los puntos ecológicos.

Consecuentemente es función de la escuela crear conciencia entre todos los miembros de la comunidad educativa donde se educan los niños y jóvenes del corregimiento, quienes de manera posterior serán agentes de cambio social y comunitario. El embellecimiento de la escuela será el punto de partida para las transformaciones de sus barrios y entorno, implementando estrategias desde la investigación para guiar un aprendizaje en contexto, que además aporte al desarrollo sostenible local.

Por, sobre todo, esto explicaría las necesidades básicas para la población no están cubiertas y en el futuro, una distribución equitativa de los recursos naturales, será imprescindible para garantizar la estabilidad social y ambiental de los moradores de Tucurinca. De manera general, de hecho, los fenómenos naturales ocasionados por el daño a los ecosistemas como inundaciones, y sequías, ocasionan escasez de alimento, pérdida a la propiedad de miles de familias y una consecuente inestabilidad social, lo cual hace aún más difícil promover la conciencia ambiental, ya que la gente en su desesperación por satisfacer sus necesidades inmediatas, tiende a agotar los recursos, impidiendo que éstos se regeneren y por lo tanto no se cumplen los objetivos del desarrollo sustentable.

Ante esta situación, las necesidades básicas para la población no están cubiertas y en el futuro, una distribución equitativa de los recursos naturales, será imprescindible para garantizar la estabilidad social y ambiental de los moradores de Tucurinca. De manera general, los fenómenos naturales ocasionados por el deterioro de los ecosistemas como inundaciones, y sequías, ocasionan escasez de alimento, pérdida del patrimonio de miles de familias y una consecuente inestabilidad social, lo cual hace aún más difícil promover la conciencia ambiental, ya que la gente en su desesperación por satisfacer sus necesidades inmediatas, tiende a agotar los recursos, impidiendo que éstos se regeneren y por lo tanto no se cumplen los objetivos del desarrollo sustentable.

Para llevar a cabo los anteriores postulados, es necesario crear en los niños y jóvenes una educación con conciencia, porque a través de ella se logra la construcción de conocimientos y formas de comportamiento para transformar el medio con la lógica de la eficiencia y los referentes de las leyes termodinámicas y ecosistema. De igual forma, mediante una formación investigativa, que apoyada por sus docentes los involucre en la resolución de problemas, en la mediación de situaciones que requieren ser atendidas o en la intervención de factores que determinen las mejoras a corto, mediano y largo plazo. Es aquí donde se hace presente el uso de objetos virtuales de aprendizaje significativos, donde los actores puedan hacer uso de las tecnologías para estar a tono con los cambios del mundo actual.

\section{Educación ambiental mediante proyectos productivos}

Cabe decir que, que los problemas ambientales están relacionados con el modelo o sistema de mejora económica y social, situación que cada vez más empeora y se torna más irreversible. El sistema de apropiación/producción y derroche actual nos está llevado a una situación crítica de la cual no será nada sencillo salir, pues 
enfatiza en la desregulación real del crecimiento económico-productivo, propio de un sistema que cosifica los ecosistemas y solo les da un valor económico, mercantil. El deterioro del ambiente se ve influenciado por un alto índice de analfabetismo, por lo que, por medio de un proceso educativo, la sociedad transmite determinados valores los cuales van moldeando el comportamiento de los estudiantes.

Por otro lado, la educación ambiental debe plantearse como un conocimiento integrado del medio socio-natural, donde la mente es un sistema activo-participativo, donde todo está en relación con todo, como un proceso abierto, flexible y creativo, para la solución de problemas socio-ambientales (Martínez, 2007). Es necesario un enfoque sistémico, donde los objetos de la realidad son sistemas organizados, integrados y jerarquizados unos con otros. Trascender la lógica, mediante el vínculo naturaleza-sociedad, con un enfoque integrado de ambas, entre ciencias naturales y sociales, donde se concreta el conocimiento: (a) valoración ambiental (no económica) del medio natural; (b) relaciones ecológicas y la participación del ser humano; (c) paisaje y sensibilización estética y conservación del medio; (d) Impacto producido por el ser humano sobre el ambiente y sus formas de producción; (e) Concienciación ante la problemática ambiental; (f) Negociación en el análisis y resolución de conflictos entre humanos; (g) Fomento de hábitos por una mejor calidad de vida; y la (h) Consumidores y usuarios activos y críticos.

La educación ambiental debe replantear, dentro del marco histórico y su relación de dominación convencional, una visión filosófica del ambiente y de la sociedad. A través de los diversos enfoques pedagógicos (didácticos) plantea dialécticamente, nuevas estrategias metodoló- gicas transdisciplinarios en los procesos de enseñanza-aprendizaje, sobre aspectos sociales y ambientales: valores ambientales, nuevos estilos de vida, más justos, equitativos y sustentables. La educación ambiental como dimensión pedagógica de las transformaciones sociales y económicas propuestas multilateralmente para el afrontamiento de las problemáticas ambientales está llamada a responder una gran demanda social, su establecimiento como campo de práctica pedagógica debe revisarse en términos de sus hallazgos, prácticas, discusiones teóricas y métodos como espacio temático de la pedagogía que darán cuenta de las transformaciones del campo.

La educación ambiental no puede estar aislada de la dinámica social contemporánea, caracterizada por diversos movimientos sociales de indignación y la emergencia progresiva de una ciudadanía cada vez más consciente de los lazos indisociables entre las realidades sociales y ecológicas y que reivindica una democracia renovada para favorecer el bien común, (Sauvé, 2014).

El enfoque de la estrategia múltiple de la educación ambiental tiene un carácter integrador y globalizador, para desarrollar una nueva visión del mundo, más social, más sensible a lo ambiental, con un sentido entrópico, sistémico, es decir holístico. La educación ambiental es transdisciplinarios, debe ser aplicada desde varias áreas disciplinarias en forma conjunta: perspectiva científica (ecología, biología, sociología, economía, política), cultural (ideología, valores, conductas, actitudes, tradiciones, espiritualidad) e integradoras (aprendizaje, recreación) (Martínez, 2009). Además, existe una relación sinérgica entre el comportamiento proambiental y el comercio justo, donde el vínculo del individuo, con el consumo 
responsable e inteligente y el comercio justo son una cadena que posibilita el desarrollo sostenible. (Cortes, 2016).

Cabe agregar, que la educación ambiental (EA) es un elemento utilizado desde las escuelas para crear consciencia en la comunidad educativa, está debe integrar conocimientos, actitudes y acciones, donde no sólo se informe sobre un determinado problema sino además encontrar respuesta o soluciones para detener y evitar el deterioro ambiental; aún más, se trata de asumir, comprender el problema y actuar sobre él. Es decir, la educación ambiental busca una conducta responsable, conocimientos sobre el medio ambiente y su problemática, además de estrategias para actuar sobre y para el medio, (Navarro, y Garrido, 2006).

La (EA) está orientada hacia la acción, esta debe involucrar a los estudiantes en el tratamiento de problemas reales y concretos, sin conformarse con la mera discusión de posibles soluciones. Se trata de prepararse para la acción ambiental, de adquirir capacidades generales, habilidades y rutinas concretas mediante estrategias de mejora del medio. Los alumnos no sólo deben saber valorar, sino estar preparados y capacitados para la acción ambiental, (Espejel, \& Flores, 2012).

Así mismo, la (EA) pretende lograr un cambio de enfoque, desempeñando un papel esencial en la comprensión y análisis de los problemas socioeconómicos, despertando conciencia y fomentando la elaboración de comportamientos positivos de conducta con respecto a su relación con el medio ambiente, poniendo de manifiesto la continuidad permanente que vincula los actos del presente a las consecuencias del futuro, (Castillo, 2011).

La (EA) está guiada bajo 4 niveles o componentes para su ejecución: iniciando por los fundamentos ecológicos; este nivel incluye la instrucción sobre ecología básica, seguido de concienciación conceptual; este consiste en mostrar cómo el conocimiento de la normatividad ambiental puede ayudar a guiar las conductas humanas, fomentando el cuidado del medio, en tercer lugar, la investigación y evaluación de problemas; este implica aprender a investigar y evaluar problemas ambientales, finalmente; la capacidad de acción; componente que enfatiza en dotar a la comunidad con habilidades necesarias para participar productivamente en la solución de problemas ambientales presentes y en la prevención de problemas ambientales futuros (Carrato, y Marval, 2007).

Además, es necesario tener en cuenta desde la economía ecológica el proceso denominado "Metabolismo social", el cual, indica que la sociedad necesita de unos recursos naturales de los que se apropia con el fin de transformarlos, hacerlos circula y a partir del uso generar desechos que son excretados a la naturaleza, lo cual se puede observan en el diseño o elaboración de las huertas escolares ecológicas, donde los estudiantes logran tomar unos recursos y transformarlos, generando un beneficio de consumo a la comunidad. (Herrera \& Bravo, 2013)

En la actualidad, existen propuestas metodológicas de enfoques basados en investigación, entendidos como la forma de materializar lo que se hace y como se implementa donde surgen infinidad de propuestas metodológicas que se basan en un enfoque o toman varios para hacer de ellos una propuesta metodológica; es el caso de las pedagogías basadas o fundadas en investigación, las cuales se desarrollan en diferentes contextos y asumen variados caminos en coherencia con los paradigmas y corrientes en los cuales se inscriben. Dentro de esta teoría basada en investigación se encuentra 
la Enseñanza por descubrimiento y el Programa Ondas, con su propuesta de la Investigación como Estrategia Pedagógica (IEP), como una perspectiva que se desarrolla en ese enfoque y toma elementos de la pedagogía critico-liberadora y del enfoque socio-cultural, (Mejía, \& Manjarrés, 2010).

Los contenidos del proceso educativo se convierten en problemas, y a partir de preguntas se estructura el currículo, en donde los avances y desarrollos de los estudiantes se determinan por la ampliación de estructura previa que se manifiesta en lenguaje, raciocinios y conocimientos. Ese descubrimiento no es necesariamente autónomo, sino que se hace guiado por el profesor, quien se encarga de planificar los ejercicios y los procedimientos para el fin buscado, (Pozo, \& Gómez, 2006).

En ese sentido, pensar la investigación desde las corrientes educativas criticas significa una lucha teórico-práctica por la manera como sus presupuestos sobre el conocimiento, la ciencia, su epistemología, la cultura, lo humano y los grupos sociales enmarcan una acción que durante cuatrocientos años ha sido señalada como objetiva, y que no solo ha construido una forma de ella, sino que también ha ayudado a generar formas de poder que en la sociedad han servido para el control y la gestación de desigualdades, y en estos tiempos, nuevas formas de acumulación y dominación, (Ortega, Peñuela, \& López, 2009). En el caso de la IEP, se parte de la pregunta del sentido común de los niños, niñas y jóvenes para reelaborarla en la perspectiva de la educación popular, que reconoce la existencia de saberes comunes y elaborados así en la negociación con los conocimientos disciplinares, y de las maneras como se correlacionan en la negociación cultural, (Mariño, 2010).
Un principio de la educación popular, en la cual la realidad se conoce para transformarla transformándose a sí mismo, y esto desde un horizonte ético que busca develar y enfrentar la segregación, la exclusión y formas de control y dominación que se produce por las múltiples manifestaciones del poder en nuestra sociedad: políticas, económicas, sociales, étnicas, en el conocimiento, de subjetividades, de género y en el ejercicio de la práctica educativa y pedagógica; construir un espíritu de emancipación humana, para no desarrollar procesos de poder que controlan ni dominan, ni permitir que otros lo realicen en su propia vida, (Torres, 2007).

La IEP postula la existencia de saberes que se mueven más en la experiencia y las actuaciones de los seres humanos, y de conocimientos que están más dados en la esfera de las disciplinas; de esta forma, postula la existencia de racionalidades diferentes al euro céntrico norteamericana, (Mignolo, 2003). En efectos, las cuales igualmente deben ser reconocidas por el ejercicio educativo, haciendo real la interculturalidad y la acuerdo cultural; este ejercicio debe darle cabida a las razones más allá de la "razón universal", para hacer visible el reconocimiento del otro y de lo otro diferente a aquello que es postulado como universal, fruto de la lógica de control y de poder, visibilizar en los diferentes su saber, su historia, su cultura, y, cuando sea el caso, reconocerlos como epistemes negadas o invisibilidades. Va a ser el ejercicio de reconocer los contextos como lugares de saber. Por ello, busca devolver al acto educativo diferencia, heterogeneidad y multiplicidad fundadas en una diversidad cultural, social y cognitiva.

Desde ahí, lo que se construye en cualquier acción educativa son relaciones so- 
ciales, en las cuales se manifiestan bajo formas pedagógicas aquello que pensamos de la sociedad. Por eso, el papel del educador es ser enseñante - aprendiente, reconociendo la manera como se forma a la vez que va formando. En este sentido, el papel del formando es activo y también forma a sus adultos acompañantes, a la vez que va aprendiendo, gestándose unas relaciones educativas desde lo diferente en la interculturalidad, (De Souza, 2001).

En el respeto y el reconocimiento de los aportes de cada uno. Por ello en la IEP, maestro y maestra se convierten en acompañantes co-investigadores, transformando su rol tradicional y reconociéndose como aprendientes en el proceso. En el caso de la IEP, se parte de la pregunta del sentido común de los niños, niñas y jóvenes para reelaborarla en la perspectiva de la educación popular, que reconoce la existencia de saberes comunes y elaborados así en la negociación con los conocimientos disciplinares, y de las maneras como se correlacionan en la negociación cultural, (Mariño, 2010).

\section{Metodología}

\section{Diseño}

La metodología del estudio se guio a partir bajo los lineamientos de la IEP, con un diseño basado en las trayectorias de indagación, articulado al plan de estudios, transversalizando así los procesos de lectura y comprensión lectora a todas las áreas del conocimiento, lo anterior con la finalidad de generar una trasformación social y educativa a través del aprendizaje en contexto, involucrando intereses, motivaciones y realidades de los estudiantes para generar conocimiento científico.

\section{Participantes}

La unidad de análisis estuvo conformada ochenta (80) estudiantes con edades comprendidas entre los catorce (14) y dieciocho (18) años, pertenecientes a los grados decimo y once de la institución educativa Tucurinca ubicada en el Municipio de Zona Bananera, Magdalena. cabe destacar que los participantes diligenciaron debidamente el consentimiento informado.

\section{Técnicas e instrumentos}

utilizando técnicas propias de la investigación cualitativa como; diario de campo y observación participante, técnicas que permitieron registrar la realidad observada, mediante el proceso de investigación realizado, además, estas permitieron sistematizar las experiencias y reflexionar sobre la práctica pedagógica.

\section{Procedimiento}

Se establecieron cinco (5) etapas que especifican la ejecución y las metas trazadas para darle solución a la pregunta problema planteada. Las etapas utilizadas se presentan a continuación.

- Etapa (1): Convocatoria y acompañamiento para la conformación de los grupos de investigación; en esta etapa se seleccionó la muestra para realizar el estudio y posteriormente se realizó la formulación de la pregunta y planteamiento del problema de investigación.

- Etapa (2): Identificación y definición de la temática de investigación, en esta etapa se realizó la fundamentación teórica, considerando: La percepción de la realidad, 
construida por toda la sociedad de acuerdo con sus experiencias, conocimientos, costumbres, relaciones, creencias, emociones, sentimientos $\mathrm{y}$, sobre todo, intereses y necesidades. El respeto por la diversidad e identidad cultural, determinante en la formación de los educadores en la educación ambiental, porque el marco cognoscitivo del educando debe de ser lo suficientemente amplio para identificar las características de todos los elementos intrínsecos en las diferentes sociedades: identidad cultural. Y el ser humano desde una perspectiva integral, porque se requiere la transformación del mundo en una relación del colectivo y el individuo, donde la suma de las partes argumenta a esa transformación social como una unidad, forma de ser y de coexistir con su entorno, en función de un nuevo humanismo mucho más crítico y tolerante.

- Etapa (3): Diseño de las trayectorias de indagación, donde se ejecutó la organización del proceso de sistematización, se seleccionaron los instrumentos de recolección de información, se estructuro el diseño metodológico y el proceso de recolección y registro de información.

- Etapa (4): Producción de saber y conocimiento, esta consistió en el análisis de información y redacción del informe de sistematización.

- Etapa (5): Apropiación social del conocimiento, se realizó la divulgación de resultados de investigación en Espacios de apropiación municipal "El maestro tiene la palabra".

\section{Resultados}

Los principales hallazgos fueron el deterioro del humedal y la proliferación de insectos y hongos de basuras en descomposición que afectan a la salud, entonces se organizaron los subgrupos de investigación se hizo un diagnóstico a través del cuestionario dirigido a la comunidad educativa y después de analizarla se empezó a trabajar para solucionar el problema; tomamos fotos, realizamos tablas y gráfico para ir señalando lo prioritario e irle dando solución.

Después se debatió entre pares para organizar el proceso de la investigación y buscar resultados positivos e ir avanzado en el reporte a toda la comunidad educativa y general a través de hipótesis mientras se obtienen los resultados finales pero se podría decir que la población en estudio progresó en el cambio que se quiere conseguir con esta investigación; esperamos que para el 2018 el porcentaje haya avanzado en general en haber transformando su característica errada de eliminar basuras a una cultura de conservar el ambiente implementando proyectos productivos ambientales, Ornamentación y embellecimiento de los espacios de la Institución, Fortalecimiento de una cultura ambiental en la comunidad educativa, Concientizar a cerca del adecuado manejo de los residuos sólidos, Fortalecimiento de la formación en investigación para la resolución de problemas, e Implementación de Proyectos productivos ambientales

\section{Conclusiones}

La educación ambiental ante la crisis económica y social que vive nuestra realidad representa una opción viable para promover cambios sociales, nuevos conocimientos, crear conciencia de participación, de equilibrio social y ecológico, de desarrollo 
sustentable y de comportamientos culturales que hagan real una sociedad más igualitaria y solidaria, con objetivos interrelacionados para moldear una nueva dimensión del ser humano.

La educación ambiental no es un simple conjunto de conocimientos, sino una actitud creativa y práctica diaria hacia la comprensión y transformación del proceso histórico, su ubicación en el cosmos, el pensamiento humano y su nivel socioeconómico y relación ambiental; pues avanza a través de la solución a los nuevos paradigmas humanísticos que buscan evolucionar y adaptarse a las nuevas circunstancias y problemas actuales, como el deterioro ambiental y social.

La concepción de la educación ambiental integral, implica no solo la esfera de lo natural; sino también los contextos social, económico, cultural y político, desde una perspectiva histórica, dialéctica, en todos los procesos. Esta visión se da cuando se considera la construcción del conocimiento y la formación de valores y actitudes para comprender el origen y desarrollo (causa-efecto) de los problemas socio-ambientales, así como los distintos niveles de responsabilidad y participación individual y colectiva, en la búsqueda de una nueva relación sociedad-naturaleza (solución).

Los crecientes casos de contaminación del suelo es la alteración del ambiente natural por la presencia de compuestos químicos, hechos por el hombre u otra fuente, asimismo, por recibir cantidades de desechos que contienen sustancias químicas tóxicas (en cualquier estado físico: sólidos, líquidos, gaseosos) incompatibles con el equilibrio ecológico; materias radiactivas, no biodegradables y peligrosos.

En esta experiencia se aprendió mucho de los compañeros ponentes a través de sus proyectos, uno de los grandes retos de la gobernación del Magdalena, es inte- ractuar con pares para así intercambiar conocimiento, innovación y experiencias con la investigación. Este espacio permitió a docentes y estudiantes socializar los resultados de una problemática que se resolvieron a través de la IE+T. Con esta experiencia obtuvimos transformación en nuestro rol decente y en nuestros estudiantes, los cuales han mostrado desde entonces más interés por innovar e indagar. A continuación, un despliegue de evidencias de espacio de formación y autoformación liderada por la universidad de la Costa donde se hace acompañamiento, y se guía en el proceso de introducción de la IEP, al currículo.

La educación ambiental requiere de estrategias que permitan al educador ambiental canalizar acciones que conduzcan a nuevas formas de relaciones (comportamiento, conducta) sustentables de la población con el ambiente natural. Estas deben estar encaminadas a la participación de la gente, de manera que puedan comprender y transformar su realidad, para actuar solidariamente en la solución de los problemas. Por consiguiente, la educación ambiental juega un papel muy importante en la generación de estrategias para solucionar los problemas socioambientales que sufren las comunidades.

La educación ambiental esta llamada a ser tributaria de esta opción ética, la liberación para la vida plena, con abundancia de recursos de vida, lo que demanda modificar las relaciones sociales y hacerlas más justas. Ello exige una opción de carácter más radical: la opción por la vida, unido a la justicia y la paz, se halla el problema del ambiente. Se trata de lograr un nuevo estilo de vida que haga consciente al ser humano que el es parte integrante del ambiente, independientemente de las diferencias ideológicas, étnicas y culturales. 
De igual forma, en el presente estudio, se buscó promover una conciencia en la comunidad, partiendo de una gestión ambiental sostenible, con una cultura basada en el respeto y responsabilidad ambiental, desarrollando acciones como: no quemar ni talar plantas, colocar la basura en los lugares apropiados, controlar el uso de los fertilizantes y pesticidas. Por otro lado, Sensibilizar a los agentes educativos en la problemática ambiental para tomar y generar conciencia de lo que está sucediendo. Al promover la educación ambiental mediante la investigación como estrategia pedagógica en la institución educativa Tucurinca de zona bananera, se debe basar en:

a. Considerar al ambiente, en forma integral, es decir, no solo los aspectos naturales, sino los tecnológicos, sociales, económicos, políticos, morales, culturales, históricos y estéticos.

b. Asumir un enfoque transdisciplinarios para el tratamiento de la dimensión ambiental, que se inspira en el contenido específico de cada disciplina para posibilitar una perspectiva sustentable.

c. Tratar la temática ambiental desde lo particular a lo general tiene como finalidad que los pueblos se formen una idea de las condiciones ambientales de otras áreas, que identifiquen las condiciones que prevalecen en las distintas regiones geográficas y políticas, además de que reflexionen sobre las dimensiones mundiales del problema ambiental para que los sujetos sociales se involucren en los diferentes niveles de participación y responsabilidad. d. Promover el conocimiento, la habilidad para solucionar problemas, la clasificación de valores, la investigación y la evaluación de situaciones, en los individuos en formación, cuyo interés especial sea la sensibilización ambiental para aprender sobre la propia comunidad.

e. Capacitar a las personas para que desempeñen un papel en la planificación de sus experiencias de aprendizaje y darles la oportunidad de tomar decisiones y aceptar sus consecuencias. La educación ambiental, más que limitarse a un aspecto concreto del proceso educativo, debe convertirse en una base privilegiada para elaborar un nuevo estilo de vida.

La educación como factor socio-cultural es el vehículo de transmisión del conocimiento y como instrumento político importante de adaptación cultural, es un mecanismo de esperanza y cambio socioambiental en el nuevo paradigma de la sustentabilidad. El desarrollo dominante no comparte de modo equitativo, ni toma en cuenta las necesidades locales, para eliminar la pobreza y el hambre debidamente.

\section{Referencias bibliográficas}

Castillo, G. (2011). Estudio de niveles de educación ambiental formal del parque Nacional Macahlilla y su zona de amortiguamiento.

Carrato, A., y Marval, R. (2007). Propuesta de un programa de educación ambiental para la conservación del agua y recolección de residuos sólidos, aplicable a las comunidades. Universidad del Oriente, Venezuela. 
Cortés Peña, O. (2016). Sustainable development in synergistic relationship with proambiental behavior and fair trade. IJMSOR, vol. 1, no. 1, pp. 54-58

De Souza, J. (2001). Manual de estilo de la lengua española. Edições Bagaço.

Espejel, A., \& Flores, A. (2012). Educación ambiental escolar y comunitaria en el nivel medio superior, PueblaTlaxcala, México. Revista mexicana de investigación educativa.

Herrera, K., \& Bravo, E. (2013). Perspectiva de la ecología en la comprensión de los comportamientos ambientales. Omnia, 19 (3), 20-30.

Mariño, G. (2010). El diálogo en la educación de jóvenes y adultos. Dos propuestas pedagógicas para implementarlo. En: El taller dialógico / la recuperación de experiencias laborales. Bogotá. OEI.

Martínez, R. (2009). Importancia de la educación ambiental. En Revista Educare, Vol. XIV, \# 1, UNA.

Martínez, R. (2007). Enfoque de la investigación cualitativa. Revista Tópicos \#144, UNA.

Mejía, M \& Manjarrés, M. (2010). La Investigación como Estrategia Pedagógica. Programa Ondas - Colciencias.

Mignolo, W. (2003). Capitalismo y geopolítica del conocimiento. Buenos Aires: Paidós

Navarro, R. y Garrido, S. (2006). Construyendo el significado del cuidado ambiental: un estudio de caso en educación secundaria, Reice. Revista electrónica iberoamericana sobre calidad eficacia y cambio en educación (España), vol. 4, núm. 1, pp. 52-70.
Novo, María. (2006). Educación ambiental. Desarrollo sostenible y globalización. Revista de Educación Ambiental, año 4 No 6, México.

Organización de las Naciones Unidas. (2012). Política Nacional de Educación Ambiental. Recuperado de https://rio20.un.org/sites/rio20. un.org/files/a-conf.216-l-1_spanish. pdf.pdf

Ortega, P., Peñuela, D \& López, D. (2009). Sujetos y prácticas de la pedagogía crítica. Ediciones El Búho. Bogotá.

Perales, F., \& Ayerbe, J. (2016). El trabajo por proyectos y por resolución de problemas en Educación Ambiental: análisis y tendencias.

Pozo, J. \& Gómez, M. (2006). Aprender y enseñar ciencia. Ediciones Morata. 5ta. Edición. Madrid.

Sandoval, M. (2012). Comportamiento sustentable y educación ambiental: una visión desde las prácticas culturales. Revista Latinoamericana de Psicología, 44(1), 181-196.

Sauvé, L. (2014). Educación ambiental y ecociudadania. Dimensiones claves de un proyecto político-pedagógico-Environmental education and eco-citizenship. Key dimensions of a pedagogical-political project. Revista científica, 1(18), 12-23.

Torres, A. (2007). La educación popular. Trayectoria y actualidad. Bogotá: El Búho. 